\title{
Editorial
}

\section{Thermodynamics Of Inorganic Systems By High Temperature Mass Spectrometry}

\author{
Miroslaw Miller ${ }^{1}$, Laszlo Bencze ${ }^{2, *}$ and R. Viswanathan ${ }^{3}$ \\ ${ }^{I}$ Wroclaw University of Technology, Department of Chemistry, 50-370 Wroclaw, Poland; \\ ${ }^{2}$ Eötvös Loránd University, Department of Physical Chemistry, H-1117 Budapest, Pázmány Péter sétány 1/A, Hungary \\ ${ }^{3}$ Fuel Chemistry Division, Chemistry Group, Indira Gandhi Centre for Atomic Research, Kalpakkam, Tamil Nadu \\ 603102, India
}

More and more modern technologies are based on the processes taking place at high and very high temperatures. Among them solid oxide fuel cell (SOFC) technology, production of the new power-efficient light sources and novel metallurgical processes (as separation of lanthanide and actinides by chemical vapour transport) should be noticed. The devices working at high temperatures, under different atmospheres and consisting of various chemicals being in a long-term contact often require application of materials of special properties. The corrosion, diffusion or vaporization processes, negligent under the ambient conditions, become the limiting factors of materials to be applied at high temperatures. It is a reason why interest of thermodynamic properties of the chemical components at high temperatures (of $1000-2000 \mathrm{~K}$ ) is recently increasing. The knowledge of the qualitative and quantitative composition of the gaseous phase is necessary for a complete description of the chemical system under aforementioned conditions. Knudsen effusion mass spectrometry became in the last decades the most powerful tool in this kind of investigations.

The Knudsen effusion mass spectrometry method consists in a mass spectrum analysis of a beam of species effusing through the effusing orifice from the Knudsen cell. An area of the orifice in comparison to the surface of vaporizing substance is so small that the thermodynamic equilibrium in the cell is not disturbed upon effusion of vapours out of the cell. Thus, the content of the qualitative and quantitative effusing molecular beam can be considered as representing the equilibrium vapour in the cell.

Knudsen cell temperatures and intensities of ion currents originating from ionisation or fragmentation of gaseous species are the quantities measured in the course of an

*Address correspondence to this author at the Eötvös Loránd University, Department of Physical Chemistry, H-1117 Budapest, Pázmány Péter sétány 1/A, Hungary; Tel: +36 1 3722500/1571; Fax: +36 13722592 ;

E-mail: bencze@chem.elte.hu investigation by Knudsen effusion mass spectrometry. Applying equations derived from the kinetic theory of gases and chemical thermodynamics to measured mass spectra, it is possible to:

- identify all gaseous species forming the equilibrium vapor over condensed phases in the Knudsen cell;

- calculate their equilibrium partial pressures in the range from generally between $10-5$ and $10 \mathrm{~Pa}$ and up to temperatures above $2500 \mathrm{~K}$;

- compute thermodynamic properties of gaseous molecules and condensed phases from the determined partial pressures and their temperature dependencies, as, for example, enthalpies and entropies of vaporization, dissociation and formation.

For the first time mass spectrometry with Knudsen effusion method was applied in 1948 by Ionov to study vaporization of alkali metal halides. This study was followed by the investigations of Chupka and Inghram as well as by Honig to study the free evaporation of carbon. Since that time, Knudsen effusion mass spectrometry, with its attractive features of high sensitivity and resolution under high vacuum conditions, has become a useful tool for the qualitative and quantitative detection of gaseous species effusing from the Knudsen cell. Almost all groups of inorganic materials were taken into investigations, such as: borides, carbides, fullerens, nitrates, sulphates, halides, metals, alloys, oxides, glasses, ceramics. Vaporization mechanism and thermodynamic data can be obtained by the use of the method.

The present issue shows state-of-art of the method and summarises recent research activities concerning investigation of materials carried out by Knudsen effusion mass spectrometry. As contributors for the issue the best researchers have been invited, active on this highly specific and unique field of science from all the world. 